\title{
Percepciones de los estudiantes frente a la satisfacción personal desde el enfoque del desarrollo humano
}

\section{Students' perceptions regarding personal satisfaction from a human development approach}

\author{
DOI: http://dx.doi.org/10.17981/cultedusoc.11.2.2020.03
}

Recibido: Noviembre 22 de 2019 Aceptado: Febrero 14 de 2020 Publicado: 09 de julio de 2020

\author{
Mónica María Álvarez Gallego \\ Universidad Católica Luis Amigó. Medellín (Colombia) \\ monica.alvarezg@amigo.edu.co \\ Ana María Montoya López \\ Universidad Católica Luis Amigó. Medellín (Colombia) \\ ana.montoyaop@amigo.edu.co
}

Para citar este artículo:

Sánchez, L., García, C. e Iguarán, A. (2020). Bienestar psicológico de jóvenes víctimas del conflicto armado. Cultura, Educación y Sociedad, 11(2). 43-56. DOI: http://dx.doi.org/10.17981/cultedusoc.11.2.2020.03

\section{Resumen}

El presente artículo tiene como objetivo comprender las percepciones de los estudiantes frente a la satisfacción personal y su relación con los procesos educativos desde un enfoque de desarrollo humano. Metodológicamente se fundamenta en el paradigma histórico hermenéutico desde una perspectiva cualitativa, a través del estudio de caso. Para la recolección de la información se trabaja con técnicas como el grupo focal, la entrevista semiestructurada y la observación no participante. Los resultados dan cuenta de cómo el desarrollo humano y la satisfacción que sienten y viven los docentes se convierte en una estrategia, no solo de mejoramiento personal, sino también a decir de los estudiantes se refleja en la calidad de los procesos educativos. Como conclusión resalta la vinculación que realizan los estudiantes entre la gestión académica, el desarrollo humano de los docentes, su evolución personal y académica con los procesos de mejoramiento continuo al interior del aula y la calidad educativa.

Palabras clave: Gestión académica; satisfacción personal; procesos educativos; desarrollo humano; calidad educativa

\section{Abstract}

This article aims to understand the students' perceptions regarding personal satisfaction and their relationship with educational processes from a human development approach. Methodologically it is based on the hermeneutical historical paradigm from a qualitative perspective, through the case study. For the collection of information, we work with techniques such as the focus group, semi-structured interview and non-participant observation. The results show how the human development and satisfaction that teachers feel and live becomes a strategy, not only for personal improvement, but also to say that students are reflected in the quality of educational processes. In conclusion, it highlights the connection that students make between academic management, the human development of teachers, their personal and academic evolution with the processes of continuous improvement within the classroom and educational quality.

Keywords: Academic management; personal satisfaction; educational processes; human development; educational quality 


\section{INTRODUCCIÓN}

Socialmente la escuela, es el espacio por excelencia donde se generan modelos y estrategias pedagógicas, para que los individuos alcancen las competencias personales, sociales y académicas en pro del desarrollo humano. El propósito es mejorar su condición inicial como base para un futuro promisorio en cuanto a su ser, hacer y estar; todo ello redunda en su calidad de vida. De este modo la escuela ha sido generadora de modelos pedagógicos para transferir no solo conocimientos sino para aprender a convivir, reconocer la diferencia, comprender el mundo y lograr estilos de vida digna; de este modo los docentes direccionan sus actividades académicas para facilitar los procesos de aprendizaje y desarrollar capacidades personales y profesionales.

No obstante, en el logro de estos estilos de vida, no solo han tomado parte las estrategias y los ejercicios pedagógicos programados e implementados por los docentes en el aula. En otros palabras, en el objetivo de construir y lograr que tanto estudiantes y egresados alcancen una vida digna, plena de desarrollo humano, no solo interviene la gestión académica, pedagógica y administrativa de docentes y directivos de las instituciones educativas, además en ellas ha intervenido el comportamiento, la actitud y aptitud de los docentes que al manifestar satisfacción, alegría y amor por lo que hacen, de una manera no direccionada e intencionada forman a sus estudiantes un ser, hacer y saber, competencias que los capacita para direccionarlos y llevarlos a superar dificultades para conseguir las metas, objetivos y el éxito propuesto, es decir un alto nivel de desarrollo humano.

De allí que es relevante, proponer un estudio que se ocupe de conocer y comprender, qué perciben los estudiantes en relación a los docentes y administrativos, en cuanto a la satisfacción personal y su desarrollo y cómo el comportamiento de estas categorías actúa como agente inhibidor o dinamizador de los procesos educativo. Al respecto la satisfacción personal del docente se concibe como una estrategia pedagógica que puede contribuir en el estudiante al desarrollo de su personalidad, incrementar su propia satisfacción y fortalecer el desarrollo humano. De allí que esta investigación las pretende comprender las percepciones de los estudiantes del grado $10^{\circ}$ y $11^{\circ}$ de la institución Educativa Marco Tobón Mejía, ubicada en el municipio de Santa Rosa de Osos, Antioquia frente a la satisfacción personal y su relación con los procesos educativos desde un enfoque de desarrollo humano.

Igualmente se devela cómo los procesos administrativos de las instituciones educativas inciden en la creación de condiciones que contribuyan al desarrollo humano en la población docente; el propósito es alcanzar niveles de satisfacción que conlleven a fortalecer los procesos educativos, así como sus comportamientos y actitudes hacía el entorno escolar.

\section{Metodología}

El estudio se orienta a considerar la opinión de los estudiantes que forman parte de los grupos de interés en atención a las categorías: satisfacción personal - procesos educativos - desarrollo humano; la interrelación categorial se aborda desde el paradigma interpretativo o hermenéutico, considerado por Arráez, Calles y Moreno (2006), como una forma 
de interpretar los textos, para comprender el todo y sus elementos. En este paradigma la comprensión es circular, en la que la forma del lenguaje es mediadora de la existencia y la experiencia hermenéutica.

El enfoque cualitativo se orienta a "comprender los fenómenos, explorándolos desde la perspectiva de los participantes en un ambiente natural y en relación con su contexto" (Hernández, Fernández y Baptista, 2014, p. 358). El método seleccionado es el estudio de caso por cuanto el estudio se centra en una situación en particular de una institución Rodríguez (1998) citado por López (2002) considera "que a través del estudio de caso se puede conseguir (...) comprobar o contrastar los efectos, relaciones y contextos presentes en una situación y/o grupo de individuos analizados” (pp. 122-123).

Con relación a las técnicas de recolección de información se emplea el grupo focal por cuanto se quiere indagar en un grupo de participantes. Según Escobar y Bonilla (2009) el grupo focal representa "una técnica de recolección de datos mediante una entrevista grupal semiestructurada, la cual gira alrededor de una temática propuesta por el investigador". (p. 52). El grupo focal lo conforman 10 estudiantes: 5 hombres y 5 mujeres del grado $10^{\circ}$ y $11^{\circ}$, del Institución Marco Tobón Mejía, ubicado en Santa Rosa de Osos, municipio del departamento de Antioquia.

\section{Resultados y Discusión}

En el siguiente apartado se presentan los resultados del estudio con su respectiva discusión; en primer lugar la percepción que tienen los participantes sobre su sentir relacionado con el quehacer en la institución; en segundo lugar, se aborda la comprensión de los estudiantes con respecto a la relación satisfacción personal - procesos académicos; finalmente, se devela la percepción de los estudiantes en torno a la satisfacción personal y cómo esta incide en su proceso de desarrollo humano.

\section{Percepción sobre la satisfacción personal}

Con base en los resultados obtenidos, en el grupo focal se logra identificar que los participantes asocian la satisfacción personal con el estado de felicidad, el cual es generado a partir de condiciones particulares, como es el caso de tener calidad en las relaciones interpersonales y realizar actividades como escuchar música o hacer deporte. A modo de ilustración, uno de los estudiantes expresa: "Sentirnos bien con lo que pasa en nuestro entorno, estar feliz, dedicar un tiempo en algo que de verdad queremos. No en algo a lo que estamos obligados, ser libres para ser felices" (P1). Al respeto y de acuerdo con Moreta, López, Gordón, Ortiz y Gaibor (2018):

La Satisfacción con la vida (SV), se enmarca en el análisis de las cogniciones y los sentimientos que tienen sobre sus vidas y la forma en como la llevan. De manera más reciente existen abundantes datos de la relación con los deseos de crecimiento y superación personal, ese deseo interno de las personas por la autoaceptación, el crecimiento como individuo, el afrontamiento de las dificultades y la superación personal (p. 114).

Otro de los participantes propone el vínculo de la satisfacción personal con el hecho de hacer ejercicio físico, de esta manera manifiesta que "a uno lo pueden tener satisfe- 
cho muchas cosas. A mi hacer ejercicios, correr, la resistencia" (P2). Este hallazgo se corresponde con los postulados de González, Huéscar y Moreno (2013), quienes expresan que "los principales factores que contribuyen a la satisfacción con la vida es la actividad físico-deportiva, la cual se asocia a la motivación, el autoconcepto, los hábitos relacionados con la salud” (p. 131). Por tanto, según los resultados, la satisfacción personal está ligada al autoconcepto y a la autoestima, aspectos que son favorecidos cuando la persona tiene hábitos relacionados con la salud.

Por otro lado, en uno de los testimonios se indica que estar satisfecho está más relacionado con el disfrute de estar acompañado especialmente sentir la compañía de la familia.

Estar con la familia, con las personas que lo rodean a uno. Como la verdad no practico ningún deporte. Yo creo que con mi mamá hemos pasado cosas como malas y buenas. Hay como un apoyo incondicional ahí, (...) es como mi mejor amiga. Es una persona incondicional en mi vida (P3).

Con relación a la familia y la satisfacción personal, Del Valle, Hormaechea y Urquijo (2015) citado por Luna, Laca y Mejía (2011), en su estudio confirma que actualmente la familia posee un significado relevante para la mayor parte de la población y que tanto los padres como los adolescentes consideran que mantener buenas relaciones familiares es un valor muy importante, situado por encima del resto de valores deseables. Fuentes citado en Luna et al. (2011), dicen que "aunque los hijos inicien durante la adolescencia nuevas e importantes relaciones con amigos y parejas, mantienen los lazos afectivos con sus padres y éstos continúan siendo una de sus principales fuentes de apego y ayuda emocional" (p.18). Esta misma visión sobre la satisfacción personal relacionada con la familia, es la percepción de otro de los entrevistados en el grupo focal l: "no sé, depende de los gustos de cada persona. Yo digo que tener una buena relación con mi familia y con las personas que me rodean" (P2). Bajo esta perspectiva, la familia juega un rol fundamental en la satisfacción personal de los estudiantes, ya que esta les brinda un apoyo integral, desde el cuidado y el cubrimiento de necesidades básicas (alimentación, vivienda, recreación, etc.) hasta la formación y el apoyo emocional.

Es relevante resaltar testimonios que conciben la satisfacción en la vida como condición subjetiva en dependencia a las visiones de cada persona; de las necesidades y expectativas de cada individuo; sin embargo. Al respecto, otro participante expone que:

Cada persona pues puede depender de cosas distintas para sentirse satisfecho, pero personalmente, como que lo que me hace sentir más plena es estar con las personas que quiero, y saber que ese cariño es mutuo o, por ejemplo, hacer cosas que me ayudan en mi propio beneficio como lo dice el compañero o dibujar, que es lo que más me gusta a mí (E1).

Frente a este tema, diversos estudios confirman que los adolescentes asignan un significado relevante a este tipo de satisfacción y bienestar. Así con respecto al bienestar subjetivo Anguas (2001) argumenta:

El BS puede concebirse como una actitud y como tal, incluye por lo menos dos de sus componentes básicos: cognición y afecto. El componente cognitivo se refiere a los aspectos racionales e intelectuales, mientras que el componente afectivo aspectos emocionales. Se ha probado también que es utilidad subdividir el componente afectivo en dos porciones: positiva y negativa (p. 165). 
Por otra parte, las indagaciones realizadas en el grupo focal arrojan respuestas relacionadas con satisfacción personal en el ámbito educativo, al respecto uno de los participantes indica:

Personalmente siento que estudiar va a fortalecer lo que llevo construyendo como persona, porque uno acá se forma en valores que le van a servir más adelante en la vida. Yo planeo tener toda la enseñanza básica, también una enseñanza superior en la universidad y planeo tener muchos conocimientos no simplemente quedarme como en lo establecido, sino que más allá como que mi meta de vida es seguir estudiando siempre, tratando de aprender (P2).

De acuerdo con Leria-Dulčič y Salgado (2016), hay una relación entre clima social-escolar y la satisfacción con la vida en estudiantes de primaria y secundaria. En este sentido, la satisfacción con la vida puede desarrollarse en el ámbito educativo, siempre y cuando los estudiantes consoliden vínculos significativos con los demás; igualmente, el hecho de ser reconocidos, respetados y que se salvaguarden su dignidad y derechos, propicia la construcción de un proyecto de vida propio.

En este orden de ideas, el hecho de que los estudiantes tengan una visión a largo plazo (meta, sueño, propósito), hace que se sientan más satisfechos con su vida, ya que como plantean Surdez, Sandoval y Lamoyi (2018), la realización personal es un elemento que se constituye como esencial para que las personas sientan que tienen un "qué y un para qué”, que las impulsan a dar lo mejor de sí y a trabajar en pro de su desarrollo integral.

Una de las dimensiones que componen la satisfacción personal es la autorrealización, en este aspecto Surdez et al. (2018) resaltan que es importante la percepción de libertad y autonomía para el desempeño de las actividades académicas, lo que posiblemente contribuye al desarrollo de la creatividad. Asimismo, promueven la satisfacción con el desarrollo integral personal, con miras positivas hacia su futuro profesional. Al respecto, uno de los estudiantes manifiesta frente a la cuestión de estudiar profesionalmente una vez culminen sus estudios de bachillerato, que:

Lo que me hace feliz es la música y pues dicen que si usted es músico usted no va a servir para nada y no es así. Porque hay personas que no son felices en la vida, están en un carro solo por ganar dinero y yo no quiero hacer eso, yo quiero ser feliz, y si eso me hace feliz no me importa, uno de cualquier manera vive (P1).

En este propósito, Urquijo (2014) menciona que la pretensión de la teoría de las capacidades es evaluar el bienestar y la libertad de la persona que realmente tiene para hacer esto o aquello, las cosas resultan de valor en el ser o hacer, de esta forma expresa:

Este es un giro significativo para pensar la sociedad, porque establece diferencias relevantes sobre las distintas demandas que deben emprender las instituciones en el interior de la sociedad para mejorar la calidad de vida de sus miembros. Pues ya no se indaga por las necesidades básicas, los bienes primarios o los recursos con que cuenta un individuo, sino por sus capacidades que le permiten llegar a hacer o ser. En últimas, se indaga por la libertad con la que cuenta una persona para alcanzar aquello que valora (p. 65).

Tal como se ha visto, la satisfacción personal se relaciona con la libertad que tiene una persona de hacer lo que le gusta. Bien sea en el deporte, el arte, la literatura, una carrera profesional, un oficio; las personas se sienten realmente satisfechas si su proyecto de vida puede verse materializado. 
Por otra parte, se vincula el concepto de satisfacción personal con las relaciones entre los estudiantes y los docentes. De acuerdo con González, Pino y Penado (2017) "hay muchos factores relacionados con la satisfacción estudiantil, de los cuales algunos términos se refieren a una misma variable (interactividad e interacción estudiante) y diferentes variables son denominadas con un mismo término (docente, instructor, o facilitador)”. (p. 246). En relación con este postulado, uno de los testimonios de los participantes expresa que "uno también aprende con la actitud del profesor ¿si me entiende? Hay profesores que son muy lúdicos al momento de ejercer digamos como una actividad (...) lo que permite adquirir un conocimiento, así como entenderse unos con otros" (P2).

Cardona y Agudelo (2007) afirman que la satisfacción personal se logra en dos ámbitos:

(...) privado y público. Desde lo privado influyen aspectos como la percepción, felicidad, satisfacción de necesidades y demás aspectos subjetivos que rara vez pueden ser cuantificados. Desde lo público, (...) es fundamental determinar el contexto cultural en el que vive, crece” (..) (p. 544).

Cabe resaltar que un elemento fundamental para la satisfacción general de los estudiantes, se vincula con sus procesos académicos, lo que lleva a sentir admiración por su docente. En este sentido, el hecho de que el docente sea abierto, tenga metodologías pedagógicas efectivas y, en general, le permita al estudiante desarrollar conocimiento, se convierte en un factor fundamental para la satisfacción personal.

Por lo anterior, se puede decir que es importante que durante el proceso enseñanza aprendizaje, éste, se dé en un ambiente adecuado y propicio para tal fin. (...) el aprendizaje se construye principalmente en el marco de las interrelaciones personales que se establecen en el contexto del aprendizaje. En otras palabras, el aprendizaje se da tanto por el tipo de relación entre el maestro y sus alumnos, por el cómo se da el proceso de la comunicación en el aula y el cómo se imparten los contenidos académicos con referencia a la realidad de la clase (García, García y Reyes, 2014, p. 284).

Ahora bien, frente a la contribución de la vida escolar en la satisfacción personal de los estudiantes, uno de ellos manifiesta:

Yo creo que lo principal es el entorno que uno se forma con los compañeros y todas las personas que va conociendo a lo largo de su vida, desde que entró por primera vez al colegio, pues desde preescolar que uno va formando un círculo social muy amplio con todas las personas que va conociendo, pues no solamente de los amigos que pueden ser los compañeros, sino también con los profesores o cualquier persona que uno pueda conocer por medio del colegio (P8).

Finalmente, en cuanto al aporte de la satisfacción personal en el nivel académico, se resalta el siguiente testimonio, "si usted llega a un salón y con el no convive su mente, va a estar pensando en que en ese salón no puede convivir, cuando usted tiene buena convivencia aprende más" (P4). Al respecto y de acuerdo con Garay, Tejada y Romero (2017).

La creación de comunidades en línea de aprendizaje, basadas en la comunicación del conocimiento y la relación entre personas con intereses comunes, se vislumbra como un proceso de aprendizaje satisfactorio para que el alumnado tome conciencia de como realiza las actividades señaladas y, de esta manera, conforme la construcción de sus entornos personales de aprendizaje (p. 1252). 
Bajo esta premisa, el rendimiento y satisfacción de estudiantes está íntimamente relacionado, ya que factores como la motivación, el trabajo en equipo, la comunicación asertiva entre estudiantes y docentes y en general, los ambientes educativos afables, constituyen aspectos indispensables para que los estudiantes puedan llevar procesos de formación que se correspondan con sus expectativas, lo cual, de manera directa posibilita que aumente su nivel de satisfacción personal. Así pues, las relaciones interpersonales que se gestan en el aula de clase entre compañeros y docentes, permiten desarrollar procesos académicos más efectivos, posibilitan aumentar el nivel de felicidad, la autoestima y, por ende, de satisfacción personal.

\section{Relación entre procesos académicos-pedagógicos y la satisfacción personal}

Resulta pertinente evidenciar la relación entre la satisfacción personal y los procesos académicos, no sin antes y de forma general exponer lo encontrado en el grupo focal con el concepto de gestión académica; constructo que los estudiantes vinculan a procesos de mejoramiento continúo al interior del aula de clase, de hecho, lo vinculan directamente con sus propias experiencias. Así lo hace saber uno de los participantes de la investigación, cuando afirma que "la gestión académica es como los cambios de profesores (...) Suena como un cambio de mejoramiento" (P5). Otra visión apunta a que "es como una evaluación procedimental tanto para estudiantes como para profesores, ahí es donde se evalúan a los estudiantes por su desempeño como también a los profesores por su esfuerzo" (P6). De acuerdo con estas apreciaciones, Surco (2018) sustenta que a partir de la evaluación se busca optimizar la productividad en el aula de clase, basándose en la eficiencia y eficacia y se orienta a la obtención de resultados en la educación.

La gestión académica, es también entendida por los participantes como una oportunidad de mejorar para, por ejemplo, no repetir un año escolar, así se evidencia en las palabras de un estudiante:

Perdí décimo, entonces en el segundo décimo yo vi esto, entonces se dio como un mejoramiento. Yo veo que uno aprende más repitiendo el mismo año, y yo al principio estaba muy negada que yo no iba para once, pero no me arrepiento porque igualmente aprendí (P7).

Si bien es cierto que la gestión académica posibilita una disminución en las tasas de fracaso escolar y deserción, este constructo no debe asociarse directamente con las condiciones y acciones individuales de los estudiantes, por el contrario, son un conjunto de acciones institucionales que contribuyen al mejoramiento de los procesos de enseñanza, acompañamientos, evaluación; se favorecen de esta manera los intereses de los educandos.

En este mismo orden y dirección, se determina la percepción de los estudiantes acerca de los procesos pedagógicos. De esta forma, se brindan las siguientes definiciones: "pedagogía es la forma en la cual el colegio nos está enseñando" (P1); "Es la gestión para que nosotros seamos críticos sociales (...) como usted tener bases, bases claras, para poder plantear una idea y eso es lo que practicamos aquí y lo que varios profesores intentan hacer" (P3). 
Para lograr lo anterior, se destaca que tanto los docentes directivos como los equipos de gestión deben estar orientados a identificar las necesidades del establecimiento, fijar metas en consecuencia, planificar de manera acorde con éstas, implementar las acciones que sean necesarias retroalimentando constantemente al proceso y coordinando acciones de evaluación y reflexión conjunta (Beltrán, 2014, p. 947).

En efecto, la gestión pedagógica es entendida por los participantes de la investigación como un conjunto de acciones cuya finalidad permiten a los estudiantes adquirir conocimientos. Dentro de las posiciones se resalta que, en la Institución Educativa, utilizan el enfoque pedagógico crítico-social; consecuentemente, los docentes posibilitan que los estudiantes sean artífices de sus propios procesos de aprendizaje, por medio de la resolución de problemas. En palabras de Beltrán (2014):

El rol protagónico que desempeñan los directivos en la gestión pedagógica curricular es relevante, ya que estos están llamados a asegurar actividades que permitan el aprendizaje continuo de todos los miembros del establecimiento, buscando la excelencia académica y mejoras sustentables en el tiempo (p. 947).

Con base en lo anterior, se consulta a los participantes si consideraban que había diferencia entre los procesos académicos y los pedagógica, los hallazgos apuntan a que los estudiantes no establecen una diferencia plausible entre estos dos conceptos, de hecho, resaltan que ambos se complementan e incluso, son lo mismo. Sin embargo, según la Universidad Estatal a Distancia de Costa Rica (UNED, 2005) "La gestión académica se define como el proceso orientado a mejorar los proyectos educativos institucionales y los procesos pedagógicos, con el fin de responder a las necesidades educativas locales y regionales" (p. 1). Por su parte, Sandó citado en Monzant y Bolívar (2016) expresa que:

La labor pedagógica se refiere a las atribuciones curriculares y administrativas del director que le permiten la posibilidad de conducir la escuela y de imprimirle dirección a las funciones pedagógicas y a otras relacionadas con la gestión administrativa, logrando la dirección del currículo y resultados de aprendizaje en los alumnos (p. 46).

De igual manera, se indaga con los participantes acerca de la manera en que docentes y directivos aportan en su formación integral. Al respecto, uno de los participantes manifiesta: "Influyendo... yo creo que influyendo a que nosotros no nos quedemos con lo que ellos mismos nos dicen acá" (P9). Esta visión se asocia con los postulados del Ministerio de Educación Nacional (República de Colombia, MEN, 2005), donde se indica que el docente es un facilitador, domina su disciplina y a través de metodologías activas, ofrece las herramientas necesarias para que los estudiantes comprendan el mundo desde diversos lenguajes, aprendan a vivir con los demás y sean productivos; por tanto, según la respuesta de algunos participantes, la gestión académica es favorecida a través de las metodologías activas e innovadoras aplicadas por el docente como parte del proceso educativo. Al respecto, otro de los participantes expone que "son complementos de los estudiantes, ¿por qué? Porque los profesores aprenden de los estudiantes y los estudiantes de los profesores" (P5). Frente a esto, el MEN (2005) resalta que:

Ser maestro, en el buen sentido de la palabra, significa ser aprendiz. La instrucción comienza cuando uno como profesor aprende del aprendiz, se pone en su lugar para poder comprender lo que él o ella comprende y la forma en que lo comprende (p. 1). 
De allí se deriva la importancia y la influencia de la capacidad que tengan los docentes de adaptarse a las características individuales de los estudiantes y aprender de ellos.

En otro de los testimonios relacionados con el tema, se indica: "si uno tiene buena acogida por los profesores, eso le va a ayudar muchísimo" (P8). Es evidente para los entrevistados que, en los procesos académicos, un factor fundamental es la buena convivencia entre docentes y estudiantes, pues a partir de las relaciones entre ambas partes el proceso de enseñanza-aprendizaje puede llevarse a cabo de manera efectiva, lo que le aportaría al logro de la satisfacción personal de los estudiantes.

Dentro de la investigación y relacionado con los procesos académicos pedagógicos, específicamente en relación con las estrategias que utiliza el docente en el aula para fortalecer las competencias del saber, ser, hacer, sobresale lo que manifiesta uno de los participantes: "yo creo que depende también el salón, el grupo que sea, porque usted utiliza algo en este salón, pero puede que no le funcione en el otro" (P1). Asimismo, otro de ellos indica, "todos tienen su método, su manera diferente, pero todo depende de las ganas de uno, de lo que uno haga" (P4). En consonancia con las anteriores manifestaciones, Martínez (2004) indica:

Dentro del salón de clases, el profesor debe tener la certeza que sus alumnos son diferentes. Dentro de esas diferencias están la forma de hablar y estructurar las ideas, la forma en que escriben y otros comportamientos asociados al contexto dentro del salón de clases. Pero otros rasgos hay que estudiarlos con más detenimiento, como sus motivaciones, necesidades, intereses, ansiedades, sus formas de aprender y modos de resolver problemas y conflictos, ya que a veces no son tan explícitos (p. 51).

De manera general, los participantes resaltan que cada docente tiene su metodología de enseñanza, sin embargo la mayoría de ellos optan por estimular un aprendizaje autodidacta en los estudiantes, lo cual les permite a estos últimos ser los precursores de su propia evolución académica. El siguiente aporte recrea un poco lo dicho:

Como dice la compañera, eh, por ejemplo, Alexa la que nos da a nosotros español, ella 'ah, vos sos bueno para redactar textos', entonces a vos te va a exigir más, por ejemplo, haciendo una tesis, usted tiene que sacar más conclusiones, ¿Quién tiene más potencial? Usted (...), haga más argumentos y lea más', entonces es como eso, es como que (...), Miren que ellos sí le dan la oportunidad de conocerlo (...) (P9).

En este testimonio se evidencia que:

El maestro del siglo XXI es un formador de ciudadanos, capaz de leer los contextos locales y globales que le rodean y de responder a los retos de su tiempo. Es un facilitador que domina su disciplina y que, a través de metodologías activas, ofrece las herramientas necesarias para que los estudiantes comprendan el mundo desde diversos lenguajes, aprendan a vivir con los demás y sean productivos (MEN, 2005, p. 2).

Dadas estas consideraciones, los participantes expresan que los docentes se enfocan en resaltar las habilidades, competencias y destrezas de los estudiantes, de esta forma se posibilitan procesos efectivos dentro de los equipos de trabajo; los mismos estudiantes pueden ser los orientadores de sus compañeros, lo cual es positivo para la calidad académica y se refuerzan los vínculos interpersonales fundamentales para la satisfacción personal de cada uno de los jóvenes. 


\section{Comprensión de los estudiantes en torno al desarrollo humano}

Frente a este tema algunos participantes destacan que el desarrollo humano está ligado con la evolución de las personas en cada etapa vital, es decir, desde recién nacidos, hasta la vejez. Así pues, la visión de desarrollo humano se vincula a una dimensión biológica, desde una perspectiva mental o cognitiva, ya que, conforme pasa el tiempo el individuo además de evolucionar en su sistema fisiológico, mejora aspectos como la madurez y la regulación emocional. Como ejemplo de lo anterior se presenta el siguiente testimonio: "Pues, desarrollo humano como en las etapas, (...) entonces pasan los años, yo tengo que caer en cuenta que ya no puedo hacer lo que hacía en el colegio, ya tengo que actuar como una persona madura" (P3). Otra de las posiciones develadas fue:

Yo diría que lograr llegar a once, o sea, uno en sexto es un gamín, uno es muy gamín entonces uno no piensa nada- vamos a montar bicicleta, con el celular- entonces ya van pasando los años, cuando uno llega a octavo va pensando que sí, la madurez (P6).

Bajo esta perspectiva, el desarrollo humano se encuentra relacionado con las habilidades emocionales que sustente un individuo. En este propósito, para Serrano y García (2010) y Akl, Pilar y Aponte (2016), las dimensiones emocionales cumplen un significativo papel funcional y estructural en el comportamiento, por ejemplo, la inteligencia emocional, es la meta-habilidad que consiste en la gestión inteligente de las emociones, e incluye las competencias emocionales de autoconocimiento, autocontrol, motivación, empatía y manejo efectivo de las relaciones sociales, las cuales se vinculan con el desarrollo humano. Las emociones en sí mismas representan estrategias de afrontamiento para empoderarse y resolver problemáticas de índole personal y social.

Los hallazgos de este estudio además sugieren que para algunos participantes, el concepto de desarrollo humano se asocia a disfrutar cada etapa de la vida:

Bueno, yo digo que uno de mis mayores logros fue como disfrutar cada momento de mi niñez, pues porque esos son momentos que prácticamente nunca volverán ¿sí me entiende?, pero lo bueno es que compartió, disfrutó y pues que se relacionó con las demás personas, además también en los cambios físicos y en los cambios como personales como la maduración, o sea, uno niño piensa como niño, uno joven piensa más diferente (P2).

Desde esta mirada, el desarrollo humano se identifica como un proceso a partir del cual las personas pueden mejorar sus competencias y potenciar sus fortalezas. Al respecto, Dongil y Cano (2014) definen desarrollo humano como:

Un proceso mediante el cual las personas intentamos llegar a acrecentar todas nuestras potencialidades o fortalezas y alcanzar nuestros objetivos, deseos, inquietudes, anhelos, etc., movidos por un interés de superación, así como por la necesidad de dar un sentido a la vida (p. 1).

La indagación realizada a los participantes sobre si se han desarrollado de manera humana y de cómo les sirve en la relación con sus compañeros, se resalta uno de los testimonios:

Aparte de sentir felicidad, pues uno va a compartir como esa emoción con otra persona, entonces uno, pues de acuerdo a las experiencias, a lo que vivió, lo que paso el fin de semana, entonces uno como que también la otra persona (P7). 
Según un boletín entregado por la Vicepresidencia de República Dominicana (2018) "el desarrollo (económico, social y humano) será tan significativo como lo perciban las personas; esto es, su impacto debe manifestarse en la satisfacción de necesidades humanas, específicamente: la felicidad" (p. 2).

En este sentido, para los participantes el desarrollo humano está vinculado directamente con la felicidad, aspecto que, a su vez se ve influenciado por elementos como la economía, las condiciones de vida, la educación y el entorno social y familiar de la persona. El testimonio que sustenta lo anterior, Dice:

Si usted se destaca en algo, por ejemplo, si es músico y si yo sé, yo puedo ayudarle (...), yo te puedo enseñar esto, tú quieres aprender a tocar un instrumento, pues yo sé tocarlo, yo te enseño a tocarlo. El conocimiento a mí me lo dieron gratis, yo también se lo puedo dar gratis. (P8).

Según se ha visto, la solidaridad y el trabajo en equipo son conceptos mencionados por los participantes al momento de referirse al desarrollo humano. En este sentido, el hecho de compartir con el otro y apoyarlo en sus procesos, se constituye en una oportunidad de desarrollo mutuo.

\section{ConClusiones}

La satisfacción personal es subjetiva y depende de las visiones, necesidades y expectativas de cada individuo, de lo que se deduce que esta categoría se puede percibir desde diferentes sentidos de la vida tanto a nivel personal como familiar, esta puede generar diversas emociones que no solo se reflejan a en la dimensión individual, sino social y académica.

La satisfacción personal se relaciona con la libertad que tiene una persona de hacer lo que le gusta. Bien sea en el deporte, el arte, la literatura, una carrera profesional, un oficio, las personas se sienten realmente satisfechas si su proyecto de vida puede verse materializado.

Los procesos pedagógicos están asociados con las actividades de mejoramiento continuo al interior del aula de clase, de hecho, lo relacionan directamente con sus propias experiencias, por ejemplo: "mejorar para no volver a repetir un año", si bien es cierto que la gestión académica posibilita que se disminuyan las tasas de fracaso escolar y deserción, este constructo no debe asociarse directamente con las condiciones y acciones individuales de los estudiantes, por el contrario, son un conjunto de acciones institucionales que, al ser desarrolladas permiten que se mejoren los procesos de enseñanza, acompañamientos, evaluación, favoreciendo de esta manera los intereses de los educandos.

Sobre la categoría de desarrollo humano, los testimonios proponen e identifican el concepto como un proceso a partir del cual las personas pueden mejorar sus competencias y potenciar sus fortalezas, sin limitarlas a un aspecto o a una condición biológica; está vinculado directamente con la felicidad, aspecto que, a su vez se ve influenciado por elementos como la economía, las condiciones de vida, la educación, el entorno social y familiar en el que se desenvuelven las persona, en otras palabras el desarrollo humano se relaciona con el contexto. Este último aspecto lleva a concebir dentro del desarrollo humano categorías como la solidaridad y el trabajo en equipo, categorías que fueron mencionados por los estudiantes que participaron del grupo focal. En este sentido, el hecho de compartir con el otro, apoyarlo en sus procesos, se constituye como una oportunidad de desarrollo mutuo y mancomunado. 


\section{REFERENCIAS}

Akl, P. M., Pilar, E. y Aponte, F. (2016). Estrategias de afrontamiento en mujeres víctimas de violencia intrafamiliar. Cultura Educación y Sociedad, 7(2), 105-121. Recuperado a partir de https://revistascientificas.cuc.edu.co/culturaeducacionysociedad/article/ view/1105

Anguas, A. (2001). Identificación y validación del significado del bienestar subjetivo en México: fundamentos para el desarrollo de un instrumento de medición. Interamerican Journal of Psychology, 35(1), 163-183. Disponible en https://journal.sipsych.org/index. $\mathrm{php/IJP/issue/view/89}$

Arráez, M., Calles, J. y Moreno, L. (2006). La Hermenéutica: una actividad interpretativa. Sapiens. Revista Universitaria de Investigación, 7(2), 171-181. Recuperado de https:// www.redalyc.org/articulo.oa?id=410/41070212

Beltrán, J. C. (2014). Factores que dificultan la gestión Pedagógica curricular de los jefes de unidades técnico pedagógicas. RMIE, 19(62), 939-961. Recuperado de https://www. comie.org.mx/revista/v2018/rmie/index.php/nrmie/article/view/213

Cardona, D. y Agudelo, H. (2007). Satisfacción Personal como Componente de la Calidad de Vida de los Adultos de Medellín. Revista Salud Pública, 9(4), 541-549. Disponible en https://pesquisa.bvsalud.org/portal/resource/pt/lil-472257

Del Valle, M., Hormaechea, F. y Urquijo, S. (2015). El Bienestar Psicológico: Diferencias según sexo en estudiantes universitarios y diferencias con población general. Revista Argentina de Ciencias del Comportamiento, 7(3), 6-13. Disponible en https://revistas. unc.edu.ar/index.php/racc/article/view/10723

Dongil, E. y Cano, A. (2014). Desarrollo Personal y Bienestar. Madrid: Sociedad Española para el Estudio de la Ansiedad y el Estrés (SEAS). Recuperado de http://www. bemocion.mscbs.gob.es/comoEncontrarmeMejor/guiasAutoayuda/docs/guia_desarrollo_personal_y_bienestar.pdf.

Escobar, J. y Bonilla, F. (2009). Grupos focales: una guía conceptual y metodológica. Cuadernos hispanoamericanos de psicología, 9(1), 51-67. Recuperado de http://biblioteca.udgvirtual.udg.mx:8080/jspui/bitstream/123456789/957/1/Gupos\%20focales\%20 una\%20gu\%c3\%ada\%20conceptual\%20y\%20metodol\%c3\%b3gica.pdf

Garay, U., Tejada, E. y Romero, A. (2017). Rendimiento y satisfacción de estudiantes universitarios en una comunidad en línea de prácticas. RMIE, 22(75), 1239-1256. Disponible en https://www.comie.org.mx/revista/v2018/rmie/index.php/nrmie/article/view/55

García, E., García, A. y Reyes, J. (2014). Relación maestro alumno y sus implicaciones en el aprendizaje. Ra Ximhai, 10(5), 279-290. Disponible en https://docplayer.es/44442764Ra-ximhai-issn-universidad-autonoma-indigena-de-mexico-mexico.html

González, G., Huéscar, E. y Moreno, J. (2013). Satisfacción con la vida y ejercicio físico. Motricidad. European Journal of Human Movimiento, 30(1), 131-151. Disponible en https://www.eurjhm.com/index.php/eurjhm/article/view/305

González, M., Pino, M. y Penado, M. (2017). Estudio de la satisfacción percibida por Los estudiantes de la UNED con su vida universitaria. RIED. Revista Iberoamericana de Educación a Distancia, 20(1), 243-260. Disponible en http://revistas.uned.es/index. $\mathrm{php} / \mathrm{ried} /$ article/view/16377/0. 
Hernández, R., Fernández, C. y Baptista, M. (2014). Metodología de la investigación. México, D.F.: McGraw-Hill / Interamericana.

Leria-Dulčič, F. y Salgado, J. (2019). Efecto del clima social escolar en la satisfacción con la vida en estudiantes de primaria y secundaria. Revista Educación, 43(1), 1-16. Disponible en https://revistas.ucr.ac.cr/index.php/educacion/article/view/30019

López, H. J. (2002). Investigación cualitativa y participativa: Un enfoque histórico-hermenéutico y critico-social en psicología y educación ambiental. Medellín: Universidad Pontificia Bolivariana.

Luna, A., Laca, F. y Mejía, J. (2011). Bienestar Subjetivo y Satisfacción con la Vida de Familia en Adolescentes Mexicanos de Bachillerato. Psicología Iberoamericana, 19(2), 17-26. Disponible en http://revistas.ibero.mx/psicologia/articulo_detalle.php?id_ volumen=2\&id_articulo=41\&id_seccion $=\&$ active $=1 \&$ pagina $=2$

Martínez, N. (2004). Las diferencias individuales y el Aprendizaje. Diálogos, 2(3), 40-47. Disponible en http://hdl.handle.net/11715/440

Moreta, R., López, C., Gordón, P., Ortiz, W. y Gaibor, I. (2018). Satisfacción con la vida, bienestar psicológico y social como predictores de la salud mental en ecuatorianos. Actualidades en Psicología, 32(124), 111-125. Disponible en https://revistas.ucr.ac.cr/ index.php/actualidades/article/view/31989

Monzant, F. y Bolívar, M. (2016). Gestión pedagógica e integración de proyectos educativos productivos en las escuelas rurales. Negotium, 13(35), 39-55. Recuperado de http:// www.revistanegotium.org/pdf/35/art3.pdf

República de Colombia. MEN. (2005). Ser maestro hoy. El sentido de educar y el oficio docente. Al tablero, (34), 5-6. [Online]. Recuperado de http://cort.as/-RgO9

República Dominicana. Vicepresidencia de la República. (2018). Bienestar, felicidad y desarrollo: Políticas sociales para el Buen Vivir. Boletín del Observatorio de Políticas Sociales y Desarrollo, 2(5), 1-2. Recuperado de http://enfoquesocial.gob.do/media/18009/ boletin-17-bienestar-felicidad-y-desarrollo.pdf.

Serrano, M. y García, D. (2010). Inteligencia emocional: autocontrol en adolescentes estudiantes del último año de secundaria. Multiciencias, 10(3), 273-280. Disponible en https://produccioncientificaluz.org/index.php/multiciencias/article/view/16826

Surco, D. (2018). Gestión Académica y Desempeño Docente, según los estudiantes de una universidad privada en Lima, Perú. Revista Industrial Data, 21(1), 83-90. http:// dx.doi.org/10.15381/idata.v21i1.14914

Surdez, E., Sandoval, M. y Lamoyi, C. (2018). Satisfacción estudiantil en la valoración de la calidad educativa universitaria. Educación y Educadores, 21(1), 9-26. Disponible en https://educacionyeducadores.unisabana.edu.co/index.php/eye/article/view/7769

UNED. (2005). Unidad 3. Visualizando la figura: gestión académica. [Online]. Disponible en http://cort.as/-RgTp

Urquijo, M. (2014). La teoría de las capacidades. EDETANIA, 46(1), 63-80. Disponible en https://revistas.ucv.es/index.php/Edetania/article/view/161

Mónica María Álvarez Gallego es profesional en Desarrollo Familiar con Especialización en docencia investigativa Universitaria. Magister en Salud Mental de la niñez y la adolescencia. https://orcid.org/0000-0002-3894-0554 
Ana María Montoya López es licenciada en Educación Preescolar de la Universidad Católica Luis Amigó (Colombia). Estuvo en calidad de auxiliar de investigación del proyecto: Satisfacción personal de los docentes y empleados y su relación con la gestión académico-pedagógica para fortalecer procesos educativos: Una mirada de los estudiantes desde el desarrollo humano. https://orcid.org/0000-0002-3298-8792 